\title{
El tratamiento con atorvastatina reduce la actividad de xantina-oxidasa unida al endotelio en pacientes con insuficiencia cardíaca crónica: ¿Un posible nuevo efecto pleiotrópico?
}

\author{
Hernán Alcaíno1, Pablo Castro2,, \\ Lorena García4, Luigi Gabrielli2 ${ }^{2}$, Guillermo Díaz-Araya3,4, José Luis Vukasovic ${ }^{5}$, \\ Iván Godoy ${ }^{2}$ y Sergio Lavandero $3,4,5$, \\ ${ }^{1}$ Facultad de Medicina, Campus San Felipe, Universidad de Valparaíso, San Felipe. Chile. \\ 2Departamento de Cardiología, Facultad de Medicina, Pontificia Universidad Católica de Chile, \\ Santiago, Chile. \\ ${ }^{3}$ Centro FONDAP Estudios Moleculares de la Célula. Universidad de Chile, Santiago, Chile. \\ ${ }^{4}$ Departamento de Bioquímica y Biología molecular, Facultad de Ciencias Químicas y \\ Farmacéuticas, Universidad de Chile, Santiago, Chile. 5 Instituto de Ciencias Biomédicas, \\ Facultad de Medicina, Universidad de Chile, Santiago, Chile.
}

Este trabajo fue financiado en parte por FONDECYT N 1010992 (P.C.) y FONDAP N 15010006 (S.L.).

\section{Resumen}

Introducción: El aumento en la actividad de la xantina-oxidasa unida al endotelio (XOec) puede participar como un importante mediador de la disfunción endotelial en la insuficiencia cardíaca crónica (IC). Las estatinas son capaces de reducir el estrés oxidativo y restaurar la disfunción endotelial a través de mecanismos independientes de la reducción del colesterol. Sin embargo, el efecto de estos fármacos en la actividad de XOec es completamente desconocido. Nosotros estudiamos la hipótesis que atorvastatina durante 8 semanas reduce la actividad de XOec de manera independiente de los cambios en el colesterol.

Metodología: Un total de 25 pacientes con IC (Fracción de eyección < 40 \% y Clase funcional NYHA II-III) recibieron placebo por 4 semanas, seguido por 8 semanas de atorvastatina $20 \mathrm{mg}$ por día. Muestras de sangre fueron recolectadas basalmente, 4 semanas y 12 semanas. La actividad de XOec y los niveles de ácido úrico fueron medidos por espectrofotometría.

Resultados: El tratamiento con atorvastatina, pero no el placebo, redujo la actividad de ecXO ( $p<0.01)$, los niveles de ácido úrico $(p<0.05)$, colesterol total $(p<0.01), L D L$-colesterol $(p<0.01)$ y

triglicéridos $(p<0.05)$ sin cambios en los niveles de HDL-colesterol y creatinina. Además, no se encontraron correlaciones estadísticas entre la fracción de cambio de XOec y las fracciones de cambio de parámetros lipídicos.

Correspondencia: Pablo F. Castro MD.

Marcoleta 367, Santiago, Chile.

Fax: (56-2) 639-2037 - Teléfono: (56-2) 354-3624.

Correo Electrónico: pcastro@med.puc.cl

Sergio Lavandero PhD. Olivos 1007, Santiago, Chile.

Fax: (56-2) 737-8920 - Teléfono: (56-2) 978-2919.

Correo Electrónico: slavander@uchile.cl 
H. Alcaíno, P.Castro, D. Greig, H. Verdejo, M. Chiong, L. García, et al.

\begin{tabular}{lcccc}
\hline Parámetro & Basal & Placebo & Atorvastatina & p \\
\hline Colesterol total, $\mu \mathrm{M}$ & $4.6 \pm 1.0$ & $4.4 \pm 1.1$ & $3.5 \pm 0.9$ & $<0.01^{*}$ \\
Colesterol LDL, $\mu \mathrm{M}$ & $2.8 \pm 0.8$ & $3.0 \pm 0.4$ & $2.4 \pm 0.2$ & $<0.01^{*}$ \\
Triglicéridos, $\mu \mathrm{M}$ & $1.8 \pm 0.5$ & $1.9 \pm 0.5$ & $1.6 \pm 0.6$ & $<0.05^{*}$ \\
Actividad XOec, & & & $<0.01^{*}$ \\
UA mL-1 min-1 & $0.047 \pm 0.1$ & $0.042 \pm 0.1$ & $0.023 \pm 0.011$ & $<0.05^{*}$ \\
Ácido úrico, mM & $0.48 \pm 0.19$ & $0.46 \pm 0.14$ & $0.31 \pm 0.13$ & \\
\hline
\end{tabular}

* significancia estadística atorvastatina versus basal y placebo.

Conclusión: El efecto beneficioso a corto plazo de la atorvastatina en relación a la mejoría de la función endotelial demostrado en estudios previos, estaría asociado a una disminución en la actividad de XOec de una manera independiente a los cambios en el colesterol, lo que sugiere la presencia de un nuevo efecto pleiotrópico de las estatinas.

\section{Treatment with atorvastatin reduces the activity of xanthine oxydase bound to endothelium in patients with chronic heart failure}

Background: An increased activity of endothelium bound xanthine oxydase (XOeb) may play an important role as a mediator of endothelial dysfunction in chronic heart failure (CHF). Statins reduce oxydative stress and improve endothelial dysfunction through mechanisms unrelated to cholesterol lowering. However, the effect of statins on XOeb activity is unknown. We hypothesized that atorvastatin administered for 6 weeks would reduce XOeb independently of changes in serum cholesterol levels.

Methods: 25 patients with CHF (NYHA class II or III with ejection fraction $<40 \%$ received placebo for 4 weeks followed by atorvastatin, $20 \mathrm{mg}$ per day, for 8 weeks. Blood samples were obtained before statin administration and 4 and 12 weeks later. Spectrophotometry was used to determine XOeb and uric aced levels. Results: Atorvastatin, but not placebo, reduced XOeb activity $(p<0.01)$, and uric acid $(p<0.05)$, total cholesterol $(p<0.01), L D L$-cholesterol $(p<0.01)$ and triglyceride levels $(p<0.05)$. No changes were observed in $H D L$ and creatinine levels. There was no correlation between XOeb changes and changes in the other lipid parameters.

\begin{tabular}{lcccc}
\hline Parameter & Basal & Placebo & Atorvastatin & p \\
\hline Total cholesterol, $\mu \mathrm{M}$ & $4.6 \pm 1.0$ & $4.4 \pm 1.1$ & $3.5 \pm 0.9$ & $<0.01^{*}$ \\
LDL cholesterol, $\mu \mathrm{M}$ & $2.8 \pm 0.8$ & $3.0 \pm 0.4$ & $2.4 \pm 0.2$ & $<0.01^{*}$ \\
Triglycerides, $\mu \mathrm{M}$ & $1.8 \pm 0.5$ & $1.9 \pm 0.5$ & $1.6 \pm 0.6$ & $<0.05^{*}$ \\
XOec activity, & & & $<0.01^{*}$ \\
UAomL-1omin-1 & $0.047 \pm 0.1$ & $0.042 \pm 0.1$ & $0.023 \pm 0.011$ & $<0.05^{*}$ \\
Uric acid, mM & $0.48 \pm 0.19$ & $0.46 \pm 0.14$ & $0.31 \pm 0.13$ &
\end{tabular}

${ }^{*}=$ statistically significant, atorvastatin vs basal or placebo.

Conclusion: The known improvement in endothelial dysfuncion related to statin use previously reported is associated to a decrease in XOec activity independently of changes in cholesterol levels, suggesting a new pleiotropic effect of statins.

Key words: endothelial dysfunction, heart failure, statins, xanthine oxydase, oxydative stress

Recibido el 28 de diciembre de 2008. Aceptado el 10 de junio de 2009 
El tratamiento con atorvastatina reduce la actividad de Xantino-oxidasa unida al endotelio en pacientes con insuficiencia...

\section{Introducción}

El estrés oxidativo juega un papel clave dentro de los mecanismos que participan en la génesis y progresión de la insuficiencia cardiaca crónica $(\mathrm{ICC})^{1}$. Evidencia reciente demuestra que el estrés oxidativo participa en los procesos de remodelado cardiovascular y disfunción endotelial, eventos asociados con mal pronóstico en la IC2-4.

En estos pacientes, Landmesser et al. han propuesto que la disfunción endotelial está relacionada, en parte, con una disminución en la actividad de la superóxido dismutasa extracelular (SODec) y a un aumento de la actividad de la xantina-oxidasa unida al endotelio o extracelular $(\mathrm{XOec})^{5}$. Mientras la SODec es uno de los sistemas de defensa enzimático antioxidante para la eliminación de especies reactivas del oxígeno (ROS), la XOec es una de las fuentes más importantes de anión superóxido $\left(\mathrm{O}^{2^{-}}\right)$en las células endoteliales vasculares 5 .

Las estatinas o inhibidores de la enzima 3-hidroxi-3-metil-glutaril CoA reductasa, son capaces de restaurar la función endotelial por varios mecanismos6-8. Uno de ellos, es la reducción del estrés oxidativo por mecanismos independientes de la reducción de colesterol6-8. En pacientes con IC, nuestro grupo y otros investigadores hemos reportado que el tratamiento con atorvastatina en un corto período de tiempo produce una mejoría en la función endotelial asociado a un aumento de la actividad de $\operatorname{SODec}^{9,10}$. Del mismo modo, las estatinas reducen la expresión y la actividad de NADPH oxidasa, una importante enzima pro-oxidante en el endotelio ${ }^{11}$. Sin embargo, si este tratamiento modifica otras enzimas pro-oxidantes, como el caso de la XOec en seres humanos, es completamente desconocido.

El presente estudio fue diseñado para investigar a corto plazo el efecto del tratamiento con atorvastatina en la actividad de XOec y, además, determinar si este efecto dependerá del cambio en el perfil lipídico en pacientes estables con IC y disfunción ventricular. Nuestra hipótesis es que la terapia con atorvastatina durante 8 semanas reduce la actividad de $\mathrm{XOec}$ a través de un mecanismo independiente a la reducción del colesterol en pacientes con ICC.

\section{Métodos}

\section{Pacientes}

Se estudiaron pacientes con IC capacidad funcional II a III de la NYHA controlados en nuestro centro universitario. Los criterios de inclusión fueron:

a) fracción de eyección ventricular izquierda medida por ecocardiograma $<40 \%$, b) tratamiento farmacológico convencional que incluye diuréticos, ß-bloqueadores, digoxina e inhibidores de la enzima convertidora de angiotensina (Tabla 1), c) estabilidad clínica durante las últimas cuatro semanas, y d) niveles plasmáticos de colesterol $<5.2$ $\mathrm{mmol} / \mathrm{L}$. Criterios de exclusión: a) síndrome coronario agudo en los últimos 6 meses, b) cirugía de revascularización o angioplastía coronaria en los últimos 6 meses; c) hipertensión arterial no controlada (presión arterial sistólica $>160 \mathrm{~mm} \mathrm{Hg}$ o presión arterial diastólica > $90 \mathrm{~mm} \mathrm{Hg}$ ); D) miocardiopatía hipertrófica y/o cardiopatía congénita; e) uso de antioxidantes o estatinas en los últimos dos meses y f) presencia de condiciones que afecten las determinaciones de estrés oxidativo, como de insuficiencia renal (creatinina plasmática $>176 \mu \mathrm{mol} / \mathrm{L}$ ), enfermedades autoinmunes, neoplasias, enfermedad pulmonar crónica y cuadros inflamatorios crónicos. No hubo modificaciones en la terapia convencional durante el estudio, la que podría alterar el estrés oxidativo y otros parámetros bioquímicos determinados. El estudio fue realizado de acuerdo con la Declaración de Helsinki de la Asociación Médica Mundial (2000) y todos los pacientes firmaron un consentimiento informado aprobado por nuestra institución y el comité de ética local. 
H. Alcaíno, P.Castro, D. Greig, H. Verdejo, M. Chiong, L. García, et al.

Tabla 1. Características clínicas de pacientes con ICC $(n=25)$

\begin{tabular}{lc}
\hline Parámetro & Basal \\
\hline Edad años & $60 \pm 14$ \\
Hombre $\mathrm{n}(\%)$ & $22(8)$ \\
Indice de Masa Corporal, Kg/m² & $27 \pm 4$ \\
FEVI, \% & $29 \pm 10$ \\
Clase Funcional NYHA, $\mathrm{n}(\%)$ & \\
II & $11(44)$ \\
III & $14(56)$ \\
Etiología, $\mathrm{n}(\%)$ & \\
Isquémica & $6(24)$ \\
No isquémica & $19(76)$ \\
Factores de Riesgo $\mathrm{n}(\%)$ & \\
Diabetes mellitus & $6(24)$ \\
Hipertensión & $18(72)$ \\
Tabaquismo & $4(16)$ \\
Creatinina, $\mu$ mol/L & $97 \pm 18$ \\
Terapia, $\mathrm{n}(\%)$ & \\
iECA & $24(96)$ \\
B-Bloqueador & $23(92)$ \\
Furosemida & $18(72)$ \\
Digoxina & $13(52)$ \\
Espironolactona & $20(80)$ \\
Aspirina o Clopidogrel & $12(48)$ \\
Hidralazina & $2(8)$ \\
Bloqueadores de calcio & $2(8)$ \\
\hline & \\
\hline
\end{tabular}

Variables continuas son expresadas como promedio \pm DS y variables categóricas como número y (porcentaje). Abreviaciones: FEVI: Fracción de eyección ventricular izquierda, LDL=Lipoptroteína de baja densidad. HDL=Lipoproteína de alta densidad. iECA= Inhibidores de la enzima convertidora de angiotensina.

\section{Diseño del estudio}

Los pacientes recibieron placebo durante cuatro semanas y, a continuación, fueron tratados con atorvastatina $20 \mathrm{mg}$ v.o. al día durante ocho semanas. Evaluaciones clínicas y bioquímicas fueron realizadas basalmente, a las cuatro y a las doce semanas. Del mismo modo, se controlaron efectos adversos clínicos a la droga, así como perfil hepático en cada control.

\section{Pruebas de laboratorio y presión arterial}

Las muestras de sangre fueron recolectadas basalmente, cuatro semanas después del trata- miento con placebo y doce semanas después del tratamiento de atorvastatina. Las muestras de plasma se obtuvieron mediante centrifugación (2000 g por $15 \min$ a $4^{\circ} \mathrm{C}$ ) y a continuación, se congelaron a $-80^{\circ} \mathrm{C}$ hasta su posterior análisis.

Para la medición de XOec, una muestra de sangre venosa (vena antecubital) se obtuvo basalmente. A continuación, un bolo de heparina (5000 UI) se inyectó en la arteria braquial del brazo (no dominante), y muestras de sangre fueron extraídas a intervalos fijos de la vena antecubital del mismo brazo $(1,3,5,7$ y 10 minutos después de 
El tratamiento con atorvastatina reduce la actividad de Xantino-oxidasa unida al endotelio en pacientes con insuficiencia...

la inyección de heparina), según lo descrito por Landmesser et al 5 La actividad de XOec se midió de acuerdo a Prajda et al. 12 y se calculó como la diferencia entre la actividad $\mathrm{XOec}$ en plasma a los 5 min. después de la inyección de heparina y basal, como se describe anteriormente 5 , y se expresó como unidades de absorbancia (UA) por $\mathrm{mL}$ de plasma por minuto.

Además, los niveles colesterol, triglicéridos, creatinina, ácido úrico ( $A U)$ y presión arterial fueron evaluados por métodos de laboratorio estándar.

\section{Análisis estadístico}

Los resultados se presentaron como promedio \pm SD para variables continuas, y como porcentaje del total de pacientes para las variables categóricas. Las variables fueron testeadas para normalidad mediante Kolmorov-Smirnov. Para la comparación de las variables continuas entre basal, placebo y atorvastatina se utilizó test de ANOVA y post-hoc de Bonferroni. Se realizó un análisis de regresión lineal de Pearson para evaluar la relación entre fracciones de cambios (definido por la diferencia entre el valor del placebo y luego de atorvastatina dividido por el valor placebo). Un valor de $p<0,05$ fue considerado significativo.

\section{Resultados}

Características basales de la población en estudio. Un total de 25 pacientes con IC fueron incorporados a este estudio. Las características basales y la terapia actual se presentan en la tabla 1.

La edad promedio fue de $60 \pm 14$ años, 22 (88\%) eran varones. La etiología de la IC fue isquémica en 6 casos y no isquémica en 19 casos. Once y 14 pacientes se encontraban en capacidad funcional II y III, respectivamente. La fracción de eyección promedio de VI fue de $29 \pm 10 \%$.

Efecto de atorvastatina en el perfil lipídico, presión arterial y niveles de creatinina plasmática Como se muestra en la tabla 2, el tratamiento con atorvastatina durante 8 semanas redujo los niveles de colesterol total $(p<0,01)$, colesterol-LDL $(p<0,01)$ $y$ triglicéridos $(p<0,05)$, sin encontrarse cambios en los niveles de colesterol-HDL, presión arterial sistólica y diastólica y creatinina. Por otro lado, el tratamiento con placebo durante 4 semanas no cambió el perfil lipídico, los parámetros hemodinámicos ni los niveles de creatinina. Además, no se registraron efectos adversos y alteraciones en las enzimas hepáticas durante el tratamiento con placebo y el uso de atorvastatina (datos no mostrados).

Tabla 2. Efecto de placebo y atorvastatina en el perfíl lipídico, presión arterial y niveles de creatinina plasmática en pacientes con ICC $(n=25)$. ${ }^{*} p<0.01$ atorvastatina vs niveles de colesterol total respecto a basal y placebo. $\dagger p<0.01$ atorvastatina vs niveles de colesterol-LDL respecto a basal y placebo. $\ddagger p<0.05$ atorvastatina vs niveles de triglicéridos respecto a basal y placebo.

\begin{tabular}{lcccc}
\hline Parametro & Basal & Placebo & Atorvastatina & p \\
\hline Colesterol total, $\mu \mathrm{mol} / \mathrm{L}$ & $4.6 \pm 1.0$ & $4.4 \pm 1.1$ & $3.5 \pm 0.9$ & $<0.01^{*}$ \\
LDL colesterol, $\mu \mathrm{mol} / \mathrm{L}$ & $2.8 \pm 0.8$ & $3.0 \pm 0.4$ & $2.4 \pm 0.2$ & $<0.01 \dagger$ \\
HDL colesterol, $\mu \mathrm{mol} / \mathrm{L}$ & $1.1 \pm 0.5$ & $1.1 \pm 0.2$ & $1.2 \pm 0.3$ & $\mathrm{~ns}$ \\
Triglicéridos, $\mu \mathrm{mol} / \mathrm{L}$ & $1.8 \pm 0.5$ & $1.9 \pm 0.5$ & $1.6 \pm 0.6$ & $<0.05 \ddagger$ \\
Creatinina, $\mu \mathrm{mol} / \mathrm{L}$ & $97 \pm 18$ & $99 \pm 21$ & $94 \pm 16$ & $\mathrm{~ns}$ \\
Presión arterial sistólica, $\mathrm{mm} \mathrm{Hg}$ & $131 \pm 16$ & $127 \pm 17$ & $128 \pm 18$ & $\mathrm{~ns}$ \\
Presión arterial diastólica, $\mathrm{mm} \mathrm{Hg}$ & $72 \pm 12$ & $73 \pm 8$ & $72 \pm 12$ & $\mathrm{~ns}$ \\
\hline
\end{tabular}


H. Alcaíno, P.Castro, D. Greig, H. Verdejo, M. Chiong, L. García, et al.

Efecto de atorvastatina sobre la actividad de XOec. Cuando se evaluó el efecto de la terapia con placebo y atorvastatina respecto a la actividad de XOec, encontramos que el tratamiento con atorvastatina, pero no el placebo, redujo signi- ficativamente la actividad de XOec (Figura 1). También se observó una disminución significativa en los niveles séricos de $\mathrm{AU}$ con el tratamiento con atorvastatina, pero no en el caso de la terapia con placebo (Figura 2).

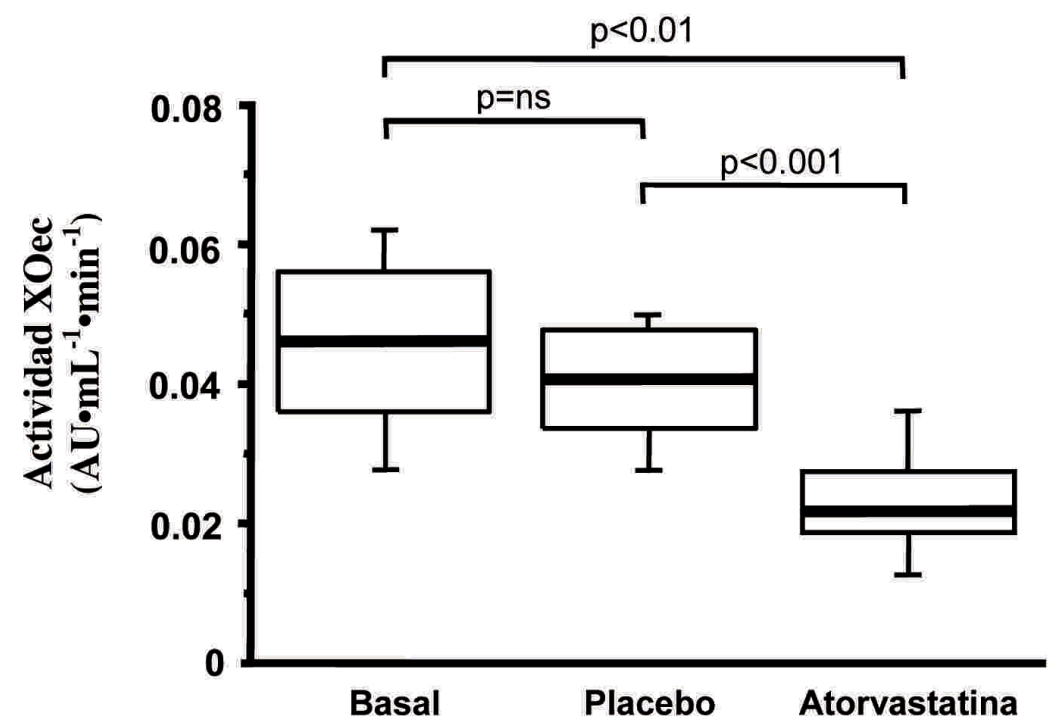

Figura 1. Efecto de atorvastatina en la actividad de xantino-oxidasa unida al endotelio (XOec) expresada como unidades de absorbancia por $\mathrm{mL}$ de plasma por minuto (UA mL-1 min-1) en pacientes con ICC $(n=25)$. La actividad de XOec basal, placebo y atorvastatina fue de $0.047 \pm 0.12,0.042 \pm 0.09$ y $0.023 \pm 0.11$, respectivamente. Los valores de significación $p$ son mostrados en la figura.

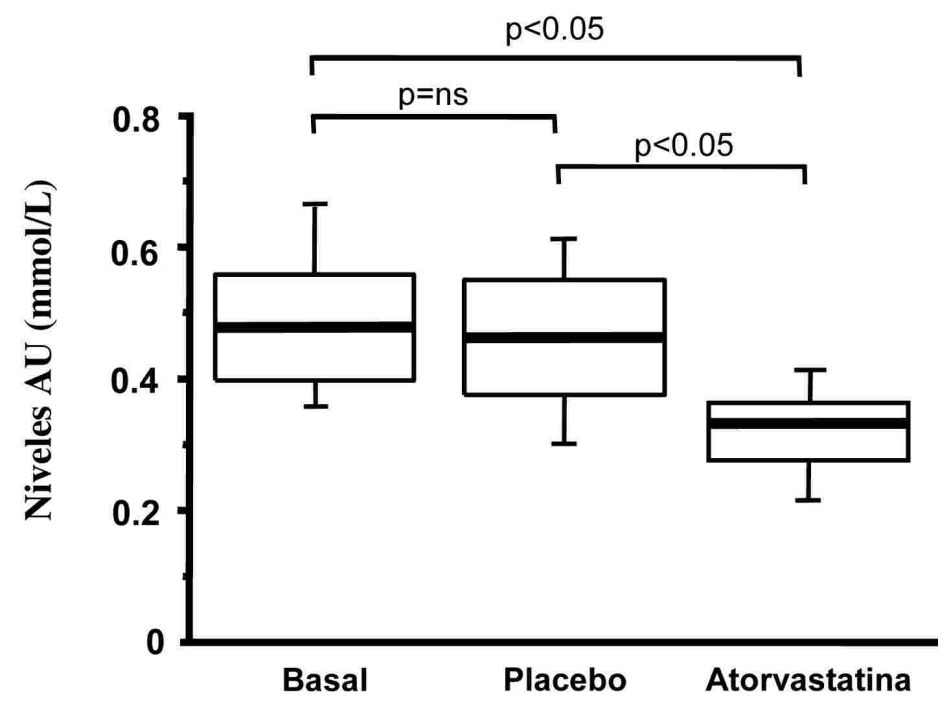

Figura 2. Efecto de atorvastatina en los niveles de $A U$ expresado como $\mathrm{mmol} / \mathrm{L}$ en pacientes con ICC $(\mathrm{n}=25)$. Los niveles basales, placebo y atorvastatina fueron de $0.48 \pm 0.19,0.046 \pm 0.14$ y $0.031 \pm 0.13$, respectivamente. Los valores de significación p son mostrados en la figura. 
El tratamiento con atorvastatina reduce la actividad de Xantino-oxidasa unida al endotelio en pacientes con insuficiencia...

Relación entre los niveles séricos de ácido úrico con la actividad de $\mathrm{XOec}$ y los niveles de creatinina plasmática después del tratamiento con atorvastatina. Se observó una significativa asociación entre la fracción de cambio del AU y la fracción de cambio de la actividad de XOec $(r=0,81, p=0,003$, Figura $3 A)$.

Por el contrario, no hubo asociación estadística entre la fracción de cambio de $A U$ y la fracción de cambio de la creatinina plasmática $(r=0,09, p=0,51$, Figura 3B).
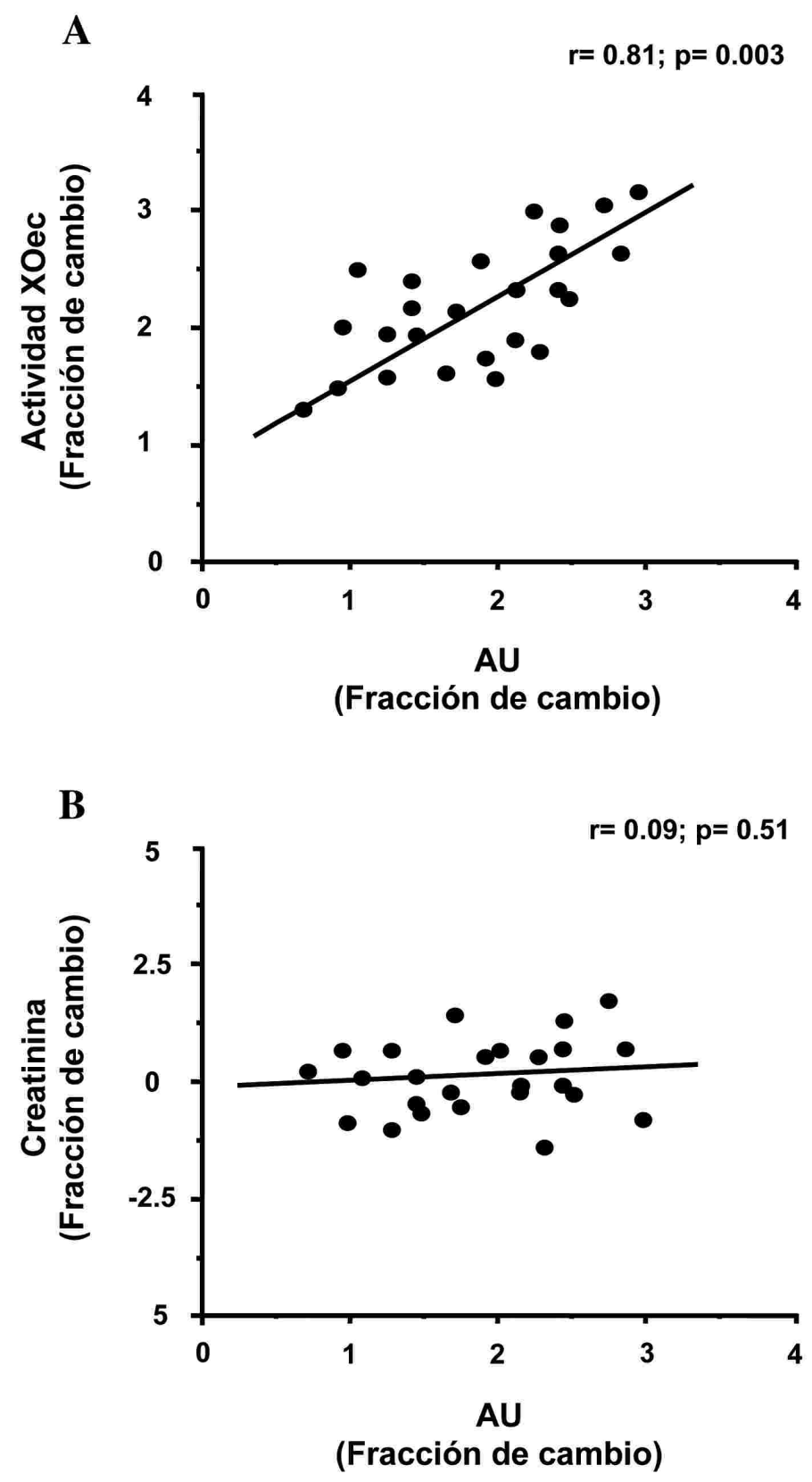

Figura 3: Correlaciones de Pearson entre: A, Fracción de cambio de los niveles de AU y la actividad de XOec B, Fracción de cambio de los niveles de AU y fracción de cambio de los niveles de creatinina después de la terapia con atorvastatina en pacientes con ICC. Los coeficientes de Pearson y sus respectivos valores de significación p son mostrados en la figura. 
Asociación entre la actividad de XOec y el perfil lipídico después de la terapia con atorvastatina

Para explorar si la reducción de la actividad de XOec estaba relacionada con el nivel de reducción de lípidos por parte de la atorvastatina, correlacionamos la fracción de cambio del perfil lipídico y la fracción de cambio de la actividad de XOec. No se observaron relaciones entre la fracción de cambio de XOec y la fracción de cambio del colesterol total $(r=0,11$, $p=0,62)$, colesterol-LDL $(r=0,08, p=0,72)$ o los triglicéridos $(r=0,15, p=0,33)$.

\section{Discusión}

Los principales resultados de este estudio fueron: 1) El tratamiento con atorvastatina, pero no el placebo, redujo la actividad de $\mathrm{XOec}$, los niveles de $\mathrm{AU}$, colesterol total, colesterol-LDL y triglicéridos, sin observarse cambios en el colesterol-HDL y función renal; 2) Existió una estrecha relación entre la fracción de cambio de AU y la fracción de cambio de la actividad de XOec y, 3) La fracción de cambio de XOec después del tratamiento con atorvastatina no mostró ninguna correlación estadística con las fracciones de cambio de parámetro lipídicos.

En los últimos años, diversos estudios clínicos en pacientes con IC han demostrado el rol de las estatinas en la prevención de eventos cardiovasculares mayores en pacientes de moderado y alto riesgo, incluso en pacientes con bajos niveles de colesterol LDL32-34. Sin embargo, los pacientes con IC han sido sistemáticamente excluidos de estos estudios haciendo alusión a problemas de seguridad en la terapia. Pese a lo anterior, existe evidencia contradictoria con respecto a la suspensión de la terapia con estatinas en pacientes con IC podría ser perjudicial35,36. Por una parte, estudios como el Controlled Rosuvastatin Multinational Trial In Heart Failure 37 (CORONA) y Gruppo Italiano Per Lo Studio Della Sopravvivenza Nell'infarto MiocardicoHeart Failure 38 (GISSI-HF) han mostrado que la terapia con rosuvastatina en pacientes con insuficiencia cardíaca isquémica y no isquémica, no confieren beneficios en cuanto a mortalidad. Estos trabajos

podrían inducir a la suspensión de la terapia con estatinas. Sin embargo, evidencia reciente en estudios poblacionales y animales han mostrado que la suspensión de la terapia con estatinas en pacientes de alto riesgo cardiovascular, particularmente en pacientes con cardiopatía coronaria, se asocian a disfunción vascular y a eventos cardiovasculares 35, 36, 39 .

Se postula que este efecto perjudicial sería secundario a la pérdida de los efectos pleiotrópicos de las estatinas y estaría mediado por mecanismos tan diversos como aumento de la actividad pro-coagulante y disminución de la actividad del óxido nítrico, entre otros $36,40$.

Diversos trabajos han sugerido que la inhibición de enzimas vasculares que producen ROS tales como $\mathrm{XO}$ y $\mathrm{NADPH}$ oxidasa, y por otro lado, un aumento de las enzimas antioxidantes vasculares pueden disminuir los eventos vasculares negativos en pacientes con enfermedades cardiovasculares, en comparación con terapias como vitaminas orales que contrarrestan a las ROS, cuyos resultados han sido desalentadores ${ }^{13-15}$.

Nuestros resultados están de acuerdo con estas observaciones. Así, un reporte reciente de nuestro grupo mostró que el tratamiento con atorvastatina reduce el estrés oxidativo sistémico (representado por el malondialdehído) y en el endotelio determinado por un aumento de la actividad de SODec9, y ahora, como se ha informado en este trabajo, una disminución en la actividad de XOec (Figura 1). Varios estudios han mostrado que la atorvastatina es capaz de disminuir los niveles de AU en pacientes con factores de riesgo cardiovascular tales como hipercolesterolemia familiar16 e hiperlipidemia primaria ${ }^{17}$ y en pacientes con enfermedad coronaria 18 .

Nuestros resultados concuerdan con estos estudios y por primera vez añadimos nuevas pruebas demostrando que el tratamiento con atorvastatina reduce significativamente los niveles de AU en pacientes con IC. Sin embargo, el mecanismo subyacente que está implicado en esta reducción es completamente desconocido.

Una posible explicación es que el efecto hipouricémico de la terapia con atorvastatina se produce principalmente por un descenso en la actividad de la XOec y no por los cambios de la función renal, si se considera 
El tratamiento con atorvastatina reduce la actividad de Xantino-oxidasa unida al endotelio en pacientes con insuficiencia...

que los niveles netos de AU presentes en la circulación periférica dependen principalmente de su producción y la capacidad de eliminación (es decir, la actividad de XO y la función renal, respectivamente). Nosotros apoyamos esta idea, porque hemos encontrado una estrecha relación entre las fracciones de cambio de XOec y los niveles de AU, no existiendo esta asociación entre las fracciones de cambio entre AU y creatinina (un parámetro de función renal). Además, nuestro grupo de pacientes tenían una función renal normal objetivada por los niveles de creatinina $<176 \mu \mathrm{mol} / \mathrm{L}$. Por lo tanto, es difícil demostrar cambios respecto a los parámetros renales. Aunque nuestro estudio se ha realizado en un corto plazo, no podemos descartar la posibilidad de que en un largo tratamiento con estatinas, la reducción de los niveles de AU pueda ser modulada más estrechamente por la función renal.

Asimismo, hemos demostrado que la atorvastatina durante ocho semanas reduce la actividad de XOec en pacientes con IC. Hasta el momento, este es el primer estudio en que se informa una disminución de la actividad de XOec con el uso de estatinas. Además, hemos demostrado que esta reducción puede ser producida de una manera independiente de los cambios en el colesterol. Nuestros resultados, a modo especulativo, podrían ser explicados a través de dos mecanismos. En primer lugar, la participación de las GTPasas pequeñas en la regulación de la actividad $\mathrm{XOec}^{19}$. Recientemente, Landmesser et al. informaron de que NADPH oxidasa regula positivamente la actividad de XOec en células endoteliales de manera redox y angiotensina II dependiente 19 . Teniendo en cuenta que la actividad de la NADPH oxidasa depende, en parte, de Rac-1, una GTPasa pequeña20,21, y porque Rac-1 es regulada negativamente por las estatinas 21,22 , nuestros datos sugieren una vía de regulación redox indirecta en la regulación de la actividad de XOec humanos con IC. En segundo lugar, es posible que exista una regulación transcripcional de la expresión y la actividad de XO. De hecho, se ha demostrado que las diversas citoquinas inflamatorias tales como el factor de necrosis tumoral- $\alpha$ (TNF- $\alpha$ ) y la interleucina-1 (IL-1) aumentan la expresión y actividad de $\mathrm{XO}$ en células endoteliales ${ }^{23}$. Debido a que las diferentes estatinas son capaces de reducir la expresión de estas citoquinas inflamatorias (TNF- $\alpha, \mathrm{IL}-1$ ) en pacientes con IC9,24-26, es posible sugerir que la atorvastatina puede reducir la actividad de $\mathrm{XOec}$ en nuestra cohorte mediante un efecto antiinflamatorio más a largo plazo.

Actualmente existe controversia si los niveles séricos de AU participan activamente en la fisiopatología de las enfermedades cardiovasculares o si sólo representa un marcador de la actividad de la XO. Hay evidencias que sugieren una acción paradójica del $\mathrm{AU}$ en el sistema cardiovascular27-28. Aunque nuestros resultados apoyan que los niveles de $A U$ sólo reflejan la actividad de XO, otros reportes de nuestro grupo sugieren al AU como un agente protector de la inactivación por el estrés oxidativo de enzimas antioxidantes vasculares, tales como la SODec ${ }^{29}$ y SOD del eritrocito (Alcaíno et al. resultados no publicados) y que están implicados en la homeostasis vascular.

Dentro de las limitaciones de nuestro estudio es posible considerar que fue un estudio abierto, se reclutó un bajo número de pacientes y la no exclusión de la terapia farmacológica estándar que podría influir en el estrés oxidativo y los niveles de $\mathrm{AU}$, como hemos informado anteriormente 30,31 . Nuestro estudio no consideró si la reducción de la actividad de XOec se relaciona con los cambios de expresión proteica. Por último, nuestras significativas correlaciones de Pearson encontradas aquí no demuestran un efecto causal de asociación, sin embargo existe evidencia experimental y clínica que pueden sustentar nuestra hipótesis. Aunque, la fracción de cambio no logró una correlación estadística con los niveles de colesterol, el escaso número de pacientes puede oscurecer esta información. En conclusión, los efectos beneficiosos de la atorvastatina en la disfunción endotelial en pacientes con ICC demostrado en estudios previos, podría estar asociado con una disminución en la actividad de XOec mediante un mecanismo independiente de la reducción del colesterol. Estos datos sugieren un nuevo efecto pleiotrópico y antioxidante de la terapia con atorvastatina. Nuevos estudios serán necesarios para investigar los mecanismos que subyacen a la regulación de la disfunción endotelial asociado al estrés oxidativo y específicamente, cómo diferentes estatinas controlan la actividad de la XOec en las células endoteliales. 


\section{Referencias}

1. PACHER P, SCHULZ R, LIAUDET L, SZABÓ C. Nitrosative stress and pharmacological modulation of heart failure. Trends Pharmacol Sci 2005; 26: 302-310.

2. FREY N. OLSON EN. Cardiac hypertrophy: the good, the bad, and the ugly. Annu Rev Physiol. 2003; 65: 45-79.

3. HEITZER T, BALDUS S, VON KODOLITSCH Y, RUDOLPH $V$, MEINERTZ T. Systemic endothelial dysfunction as an early predictor of adverse outcome in heart failure. Arterioscler Thromb Vasc Biol. 2005; 25: 1174-1179.

4. MORGAN DR, DIXON LJ, HANRATTY CG, HUGHES SM, LEAHEY WJ, ROONEY KP, et al. Impaired endotheliumdependent and -independent vasodilation in elderly patients with chronic heart failure. Eur J Heart Fail. 2004; 6: 901-908.

5. LANDMESSER $U$, SPIEKERMANN S, DIKALOV S, TATGE $\mathrm{H}$, WILKE R, KOHLER $\mathrm{C}$, et al. Vascular oxidative stress and endothelial dysfunction in patients with chronic heart failure: role of xanthine-oxidase and extracellular superoxide dismutase. Circulation 2002; 106: 3073-3078.

6. LIPINSKI MJ, ABBATE A, FUSTER V, VETROVEC GW. Drug insight: statins for nonischemic heart failure-evidence and potential mechanisms. Nat Clin Pract Cardiovasc Med. 2007; 4: 196-205.

7. LIAO JK, LAUFS U. Pleiotropic effects of statins. Annu Rev Pharmacol Toxicol. 2005; 45: 89-118.

8. MASON JC. Statins and their role in vascular protection. Clin Sci. 2003; 105: 251-266.

9. CASTRO PF, MIRANDA R, VERDEJO HE, GREIG D, GABRIELLI LA, ALCAINO $\mathrm{H}$, et al. Pleiotropic effects of atorvastatin in heart failure: role in oxidative stress, inflammation, endothelial function, and exercise capacity. J Heart Lung Transplant. 2008; 27: 435-441.

10. LANDMESSER U, BAHLMANN $F$, MUELLER $M$, SPIEKERMANN S, KIRCHHOFF N, SCHULZ S, et al. Simvastatin versus ezetimibe: pleiotropic and lipid-lowering effects on endothelial function in humans. Circulation. 2005; 111: 2356-2363.

11. RUECKSCHLOSS U, GALLE J, HOLTZ J, ZERKOWSKI HR, MORAWIETZ $\mathrm{H}$. Induction of $\mathrm{NAD}(\mathrm{P}) \mathrm{H}$ oxidase by oxidized low-density lipoprotein in human endothelial cells: antioxidative potential of hydroxymethylglutaryl coenzyme A reductase inhibitor therapy. Circulation. 2001; 104: 1767-1772.

12. PRAJDA N, WEBER G. Malignant transformation-linked imbalance: decreased xanthine oxidase activity in hepatomas. FEBS Lett. 1975; 59: 245-249.

13. HAMILTON CA, MILLER WH, AL-BENNA S, HAMILTON CA, MILLER WH, AL-BENNA $S$, et al. Strategies to reduce oxidative stress in cardiovascular disease. Clin Sci. 2004; 106: 219-234.

14. GUZIK TJ, HARRISON DG. Vascular NADPH oxidases as drug targets for novel antioxidant strategies. Drug Discov Today. 2006; 11: 524-533.

15. TAKIMOTO E, KASS DA. Role of oxidative stress in cardiac hypertrophy and remodeling. Hypertension. 2007; 49: 241-248.

16. MARAIS AD, FIRTH JC, BATEMAN ME, BYRNES P, MARTENS C, MOUNTNEY J. Atorvastatin: an effective lipidmodifying agent in familial hypercholesterolemia. Arterioscler Thromb Vasc Biol. 1997; 17: 1527-1531.

17. MILIONIS HJ, KAKAFIKA AI, TSOULI SG, ATHYROS VG, BAIRAKTARI ET, SEFERIADIS KI, et al. Effects of statin treatment on uric acid homeostasis in patients with primary hyperlipidemia. Am Heart J. 2004; 148: 635-640.

18. ATHYROS VG, ELISAF M, PAPAGEORGIOU AA, SYMEONIDIS AN, PEHLIVANIDIS AN, BOULOUKOS VI, et al. Effect of statins versus untreated dyslipidemia on serum uric acid levels in patients with coronary heart disease: a subgroup analysis of the GREek Atorvastatin and Coronary-heart-disease Evaluation (GREACE) study. Am J Kidney Dis. 2004; 43: 589-599.

19. LANDMESSER U, SPIEKERMANN S, PREUSS C, SORRENTINO S, FISCHER D, MANES C, et al. Angiotensin II induces endothelial xanthine oxidase activation: role for endothelial dysfunction in patients with coronary disease. Arterioscler Thromb Vasc Biol. 2007; 27: 943-948.

20. WANG CY, LIU PY, LIAO JK. Pleiotropic effects of statin therapy: molecular mechanisms and clinical results. Trends Mol Med. 2008; 14: 37-44.

21. ENDRES M, LAUFS U. Effects of statins on endothelium and signaling mechanisms. Stroke. 2004; 35: 2708-2711.

22. MAACK C, KARTES T, KILTER H, SCHÄFERS HJ, NICKENIG $\mathrm{G}$, BÖHM M, et al. Oxygen free radical release in human failing myocardium is associated with increased activity of rac1-GTPase and represents a target for statin treatment. Circulation. 2003; 108: 1567-1574.

23. BERRY CE, HARE JM. Xanthine oxidoreductase and cardiovascular disease: molecular mechanisms and pathophysiological implications. J Physiol. 2004; 555: 589-606.

24. MARTIN JH, KRUM H. Statins and clinical outcomes in heart failure. Clin Sci. 2007; 113: 119-127.

25. TOUSOULIS D, ANTONIADES C, BOSINAKOU E, KOTSOPOULOU M, PITSAVOS C, VLACHOPOULOS C, et al. Effects of atorvastatin on reactive hyperemia and inflammatory process in patients with congestive heart failure. Atherosclerosis. 2005; 178: 359-363.

26. MOZAFFARIAN D, MINAMI E, LETTERER RA, LAWLER RL, MCDONALD GB, LEVY WC. The effects of atorvastatin $(10 \mathrm{mg})$ on systemic inflammation in heart failure. Am J Cardiol. 2005; 96: 1699-1704.

27. SAUTIN YY, JOHNSON RJ. Uric acid: the oxidant-antioxidant paradox. Nucleosides Nucleotides Nucleic Acids. 2008; 27: 608-619. 
28. LIPPI G, MONTAGNANA M, FRANCHINI M, FAVALORO EJ, TARGHER G. The paradoxical relationship between serum uric acid and cardiovascular disease. Clin Chim Acta. 2008; 392: 1-7.

29. ALCAINO H, GREIG D, CHIONG M, VERDEJO H, MIRANDA $R$, CONCEPCION R, et al. Serum uric acid correlates with extracellular superoxide dismutase activity in patients with chronic heart failure. Eur J Heart Fail. 2008; 10: 646-651.

30. CASTRO P, VUKASOVIC JL, CHIONG M, DÍAZ-ARAYA G, ALCAINO $\mathrm{H}$, COPAJA $M$, et al. Effects of carvedilol on oxidative stress and chronotropic response to exercise in patients with chronic heart failure. Eur J Heart Fail. 2005; 7: 1033-1039.

31. CASTRO PF, DÍAZ-ARAYA G, NETTLE D, CORBALÁN R, PÉREZ O, NAZZAL C, et al. Effects of early decrease in oxidative stress after medical therapy in patients with class IV congestive heart failure. Am J Cardiol. 2002; 89: 236-239.

32. Heart Protection Study Colaborative Group. MRC/BHF Heart Protection Study of cholesterol lowering with simvastatin in 20,536 high-risk individuals: a randomised placebo-controlled trial. Lancet 2002; 360: 7-22.

33. CANNON CP, BRAUNWALD E, MCCABE CH, RADER DJ, ROULEAU JL, BELDER R, et al. Pravastatin or atorvastatin evaluation and infection therapy-thrombolysis in myocardial infarction 22 investigators. N Engl J Med 2004; 350:1495-1504.

34. WATERS DD, GUYTON JR, HERRINGTON DM, MCGOWAN MP, WENGER NK, SHEAR C. Treating to New Targets (TNT) Study: does lowering low-density lipoprotein cholesterol levels below currently recommended guidelines yield incremental clinical benefit?. Am J Cardiol 2004; 93: 154-158.

35. STONE N. Stopping statins. Circulation 2004; 110: 2280-82.

36. CUBEDDU L, SEAMON M. Statins withdrawal: clinical implications and molecular mechanisms. Pharmacotherapy 2006; 26: 1288-1296.

37. KJEKSHUS J, APETREI E, BARRIOS V, BÖHM M, CLELAND JG, CORNEL JH, et al. Rosuvastatin in Older Patients with Systolic Heart Failure. N Engl J Med 2007; 357: 2248-61.

38. TOGNONI G, POOLE-WILSON P. Effects of statins in patients with symptomatic chronic heart failure: GISSI-HF results European Congress Of Cardiology (ESC), 2008. Presentación oral.

39. HEESCHEN C, HAMM CW, LAUFS U, SNAPINN S, BÖHM M, WHITE HD. Withdrawal of statins increases event rate in patients with acute coronary syndromes. Circulation 2002; 105:1446-52.

40. CASTEJON A, ZOLLNER E, TRISTANO A, CUBEDDU L. Upregulation of angiotensin II-AT1 receptor during statin withdrawal in vascular smooth muscle cells. J Cardiovasc Pharmacol 2007; 50: 708-11. 